\title{
La Grammaire Sanskrite Étendue - État des lieuX
}

\author{
Émilie Aussant \\ UMR 7597 Histoire des théories linguistiques (CNRS - Paris Diderot - \\ Paris Sorbonne nouvelle), Paris, France
}

La présente livraison d'Histoire Épistémologie Langage se propose de prolonger, en élargissant leur horizon, les travaux pionniers initiés par Sylvain Auroux sur la grammatisation $^{1}$ massive des langues du monde à partir de la tradition grammaticale gréco-latine (cf. Auroux 1992). La grammatisation des vernaculaires européens et - aujourd'hui encore - de nombreuses langues «exotiques», sur la base de la description grammaticale élaborée pour le latin (elle-même issue d'un transfert du modèle grec), constitue un facteur d'unification théorique qui n'a certes pas d'équivalent dans l'histoire des sciences du langage (Auroux 1994, p. 82). L'histoire a néanmoins connu d'autres « grammaires étendues 》 (cf. Auroux 1992, p. 19): le modèle grammatical élaboré pour l'arabe a été utilisé pour décrire le copte, l'hébreu, le kurde, le persan, le syriaque, le turc; des outils développés pour le chinois ont été étendus au coréen, au japonais et au vietnamien; le modèle grammatical élaboré pour le grec a été utilisé pour décrire l'arménien, le géorgien, le latin, le vieux slave d'église et le syriaque; des descriptions grammaticales élaborées pour le sanskrit ont servi à décrire plusieurs langues d'Asie (variétés d'indo-aryen moyen et moderne, langues dravidiennes, vieux javanais, cingalais), une langue de Sibérie (bouriate) et des langues parlées bien loin de l'Asie (langues algonquiennes). Ce numéro d'Histoire Épistémologie Langage porte sur ce que nous conviendrons d'appeler la Grammaire Sanskrite Étendue, c'est-à-dire la grammatisation de diverses langues sur la base de descriptions grammaticales initialement élaborées pour le sanskrit.

Dans sa «Note préliminaire» à l'article d'Ojihara (1971), Colette Caillat nous rappelait l'invitation de Louis Renou «à déterminer exactement comment, et dans quelle mesure, les descriptions anciennes du sanskrit ont été imitées par les

1 Sylvain Auroux définit le concept de grammatisation comme suit (1992, p. 28): «Par grammatisation, on doit entendre le processus qui conduit à décrire et à outiller une langue sur la base des deux technologies, qui sont encore aujourd'hui les piliers de notre savoir métalinguistique : la grammaire et le dictionnaire.» 
docteurs des écoles grammaticales palies et prakrites dans les traités qu'ils ont consacrés au moyen-indien ». Près de 40 ans après cette «Note», à l'occasion d'un atelier organisé à Paris par Jean-Luc Chevillard et moi-même, historiens de la linguistique et indianistes décidèrent de travailler de concert sur la Grammaire Sanskrite Étendue. Le 3 novembre 2016 eut lieu une autre étape collective de ce projet : une journée d'étude, organisée en partenariat avec le LabEx EFL (opération EM1c « Grammaires étendues »), à Paris, réunissant sept contributeurs. Sont réunis ici cinq articles issus de communications présentées lors de cette journée.

L'atelier de 2016 s'est ouvert sur un état des lieux des recherches menées sur la Grammaire Sanskrite Étendue. On peut classer ces recherches en quatre catégories :

- les travaux menés sur la grammatisation des langues parlées en Inde (variétés d'indo-aryen moyen et moderne, langues dravidiennes, persan);

- les travaux relatifs à l'influence de modèles descriptifs du sanskrit sur les langues tibétaines;

- les travaux relatifs à l'influence de modèles descriptifs du sanskrit sur les langues d'Asie du Sud-Est;

- les travaux relatifs à l'influence de modèles descriptifs du sanskrit sur la linguistique occidentale ${ }^{2}$.

Les exemples que je mentionnerai dans cette présentation sont issus des deux premières catégories de travaux.

Sur la base des recherches réalisées jusqu'à présent, on peut commencer à dégager les grands traits qui caractérisent le transfert de descriptions élaborées pour le sanskrit à des langues autres que le sanskrit et leur adaptation à ces langues. Il s'agit, rappelons-le, d'un travail en cours, qui s'inscrit sur le long terme. Les questionnements sont encore nombreux.

\section{LES OBJETS DU TRANSFERT}

Ce qui a été transféré ou étendu sur la base de descriptions grammaticales initialement élaborées pour le sanskrit, peut se ramener à quatre catégories, qui ne s'excluent pas. Il peut s'agir:

- d'éléments relevant du champ métalinguistique: métarègles ou devises métalinguistiques, termes techniques grammaticaux;

- d'éléments relevant du domaine conceptuel : notions, idées, raisonnements;

- d'éléments relevant de la méthode ou de l'approche: descriptive, synchronique, dérivationnelle, etc.;

2 Cette dernière catégorie est l'occasion de rappeler que les descriptions grammaticales élaborées en Occident (fondées sur le modèle gréco-latin, principalement) ont également été étendues aux langues parlées en Inde (voir la contribution de Jean-Luc Chevillard). 
- d'éléments relevant de la structure ou du plan: corpus de règles (sütra) et appendices associés (catalogues des sons, des racines, de bases nominales), les sections, leur nombre, leur organisation et leurs éventuelles subdivisions, l'ordre des règles au sein des sections.

\subsection{Exemple de transfert métalinguistique}

Un exemple de ce type de transfert nous est fourni par deux traités de la tradition grammaticale tibétaine, le Sum-cu-pa («Les trente vers», SCP ci-après) et le Rtags-kyi-’jug-pa («Le guide des signes», TKJ ci-après), deux courts textes versifiés dont la datation $\left(\mathrm{VII}^{\mathrm{e}}-\mathrm{IX}{ }^{\mathrm{e}} \mathrm{s}\right.$. ?) et la paternité posent problème (Miller 2000 ; Verhagen 2000a). Verhagen (2000b, p. 211, 2001, p. 229-230, p. 233-235) indique que ces deux textes recourent ponctuellement à l'ellipse (anuvrtti en sanskrit), c'est-à-dire à la récurrence tacite, dans des règles subséquentes, d'un ou de plusieurs éléments employés dans une règle. Verhagen (2001, p. 229) donne l'exemple suivant: un groupe de règles du SCP décrit les particules du tibétain et sélectionne, à partir d'un sous-ensemble de consonnes (rjes-'jug), les constituants de ces particules. Ce sous-ensemble de consonnes est introduit dans la règle SCP 4, qui stipule que les dix consonnes qui s'emploient en position post-vocalique dans la syllabe sont $g, \dot{n}, d, n, b, m$, ', $r, l, s$. Les règles $\mathrm{SCP} 5,7,8,9,13,14$ et 15 réfèrent explicitement à ce sous-ensemble par la phrase rjes-'jug-yi-ge-bcu; les règles SCP 10-12 et 18-21, en revanche, bien que tout autant concernées par le sous-ensemble de consonnes, ne le mentionnent pas: dans ces règles, une phrase telle que rjes'jug-yi-ge-bcu-po-las «parmi les dix consonnes du sous-ensemble rjes-'jug» doit être supplée par anuvrtti, par récurrence tacite. Verhagen mentionne d'autres cas d'ellipse - dont certains sont bien plus spectaculaires - et explique que, tout comme dans les traités grammaticaux du sanskrit, le recours à l'ellipse, dans le SCP et le TKJ, a des conséquences directes sur l'ordre des règles.

Les contributions figurant dans ce numéro fournissent d'autres exemples de transferts métalinguistiques.

\subsection{Exemple de transfert conceptuel}

On peut mentionner le cas de l'adoption d'une classification sémantique de l'objet grammatical (karman), initialement formulée par Bhartrihari ( $\mathrm{v}^{\mathrm{e}} \mathrm{s}$.), un grammairien - et philosophe - (brahmane) de l'école pāṇinéenne, par Cēnāvaraiyar (XIII $\mathrm{XIV}^{\mathrm{e}} \mathrm{s}$.), un commentateur tamoul du Collatikāram («Livre des mots») du Tolkāppiyam $^{3}$, l'ouvrage fondateur de la tradition grammaticale tamoule (III ${ }^{\mathrm{e}} \mathrm{s}$. av. n. ère $-v^{e} s$.). Dans un article très détaillé de 2013, Vincenzo Vergiani explique

3 Pour plus de détails sur cet ouvrage, voir la notice n4351 du CTLF (http://ctlf.ens-lyon.fr) par Chevillard. 
comment et pourquoi Cēnāvaraiyar s'est approprié le concept d'objet grammatical et l'a adapté aux besoins de la description grammaticale du tamoul, telle qu'elle s'exprime dans le Tolkāppiyam. Pour résumer, on peut dire que, à partir de Pāṇini, la description du sanskrit distingue les catégories sémantico-syntaxiques (c'est-àdire les notions d'agent, d'objet, d'instrument, de lieu, etc.) des formes linguistiques qui les marquent (les désinences casuelles). Pāṇini distingue trois types d'objets : 1) ce que l'agent désire le plus atteindre (ipsitatama, ex. patram likhati «il/elle écrit une lettre»), 2) ce que l'agent ne désire pas atteindre mais qui est lié à l'action comme le type précédent (anīpsita, ex. caurān paśyati «il/elle voit des voleurs »), 3) ce qui n'est pas désigné autrement, i.e., par une autre notion (akathitam ca, c'est le cas de l'objet secondaire dans des constructions à double accusatif: mānavakam dharmam śāsti «il/elle enseigne la loi à l'élève»). Quelques siècles après la composition de l'Așțādhyāyz, Bhartṛhari reprend cette catégorisation et l'affine, aboutissant à sept classes de karman («objet») : le premier type d'objet identifié par Pāṇini est subdivisé en trois sous-types: «à produire » (nirvartya), «à modifier» (vikārya), «à obtenir» (prāpya), le deuxième type d'objet identifié par Pāṇini est subdivisé en deux sous-types: un objet « indifférent» (udāsina) et un objet «non désiré »(anīpsita), le troisième type d'objet identifié par Pāṇini est conservé tel quel mais un dernier type est ajouté : un objet ayant reçu auparavant une autre désignation (anyapūrvaka), ainsi, dans gacchati devadatto grāmam, devadatta- est agent, mais si l'on passe à une construction causative yajñadatto devadattam grāmam gāmayati, devadatta- n'est plus agent mais objet (il devient alors «objet ayant reçu auparavant une autre désignation $»)$.

De ces sept catégories, Cēnāvaraiyar ne reprend que les trois premières («à produire », «à modifier », «à obtenir ») : la distinction entre īpsitatama et anīpsita n'est pas évoquée, quant aux deux catégories akathitam et anyapūrvaka, elles ne sont pas pertinentes en tamoul, les structures morpho-syntaxiques correspondantes n'existant pas. On a bien affaire à un transfert du sanskrit vers le tamoul, même si le résultat est différent du modèle de départ (voir la contribution de Victor D'Avella pour un exemple similaire-karumak-karuttā).

Nous aurons l'occasion de revenir sur l'appropriation des objets transférés ciaprès $(\S 2.1)$.

\subsection{Exemple de transfert méthodologique}

Les grammaires de l'indo-aryen moyen (ou moyen-indien) nous fournissent un exemple particulièrement intéressant (voir la contribution de Nalini Balbir).

La quasi-totalité des grammaires prakrites rédigées en sanskrit ont la particularité de ne pas décrire l'intégralité de la langue mais seulement les traits qui la différencient d'une autre langue, autre langue que le descripteur considère 
comme étant sa «base» (prakrti $)^{4}$. Ces grammaires incomplètes - on peut également dire indirectes - suivent la même méthode de description, que l'on peut décrire brièvement comme suit ${ }^{5}$ :

1) les opérations enseignées permettent d'obtenir des formes prakrites en modifiant des formes sanskrites ; cette modification se manifeste, dans la plupart des cas, par une substitution: «tel phonème (en prakrit) se substitue à tel phonème (en sanskrit)» ou « il y a amuïssement - i.e., substitution par zéro - (en prakrit) de tel phonème (en sanskrit)»; notons que cette opération de susbtitution peut également porter sur des suffixes mais aussi sur des unités plus grandes (Prākrtaprakāśa - PP ci-après - de Vararuci, III ${ }^{\mathrm{e}}-\mathrm{V}^{\mathrm{e}} \mathrm{s}$.) : PP 4.21 «o se substitue [aux prépositions] ava et apa [en composition]», PP 7.15 «genha- se substitue à grah[«saisir»]»; les grammairiens recourent également à la substitution pour indiquer qu'une seule forme prakrite correspond à deux formes sanskrites, par ex. PP 6.63 « le pluriel se substitue au duel» (i.e., il n'y a plus que singulier et pluriel en prakrit) et PP 6.64 «le génitif se substitue au datif» (i.e., il n’y a plus de datif en prakrit);

2) les grammaires se terminent généralement sur le principe selon lequel tout ce qui n'a pas été traité dans ce qui précède est à inférer à partir de la base. Dans les grammaires prakrites qui décrivent plusieurs variétés (telle le Prākrtānuśāsana de Puruṣottama, fin du XI ${ }^{\mathrm{e}}$ début du XIII ${ }^{\mathrm{e}} \mathrm{s}$.), on observe que, dans l'ensemble (ce n'est pas systématique), chaque variété traitée constitue la base de la variété qui est traitée ensuite; on a donc une sorte de « description » grammaticale en entonnoir, qui procède par limitations successives : on part du plus général ou de ce qui est commun au plus grand nombre (i.e., les règles formulées pour le sanskrit, supposées connues), puis on limite la portée de ces règles à la māhārāṣțrī en décrivant seulement ce qui change par rapport au sanskrit. La portée de la « description » de la māhārāṣțrī est à son tour limitée à celle de la śaurasen̄̄, ainsi de suite.

Les grammaires incomplètes ou indirectes des prakrits fonctionnent donc comme des appendices contrastifs qui se greffent à la grammaire, complète ou directe - et supposée connue - de leur «base».

Ce type d'extension ou de transfert est tout à fait singulier : il a été conçu, non comme une reproduction de la méthodologie du modèle de départ, mais comme un prolongement de cette méthodologie. Je pense que ce qui a rendu possible cette conception, c'est la perspective générative que Pāṇini a adoptée pour

$4 \mathrm{Il}$ a tout de même existé des grammaires de langues moyen-indiennes complètes, i.e. décrivant la phonétique de la langue, la morphologie nominale et verbale et les indéclinables; c'est le cas des grammaires du pali, rédigées en pali (qui traitent aussi de l'emploi des cas, de la composition et de la dérivation) et de la section consacrée à l'apabhramśa dans le Śabdānuśāsana («Enseignement sur les mots») de Hemacandra, rédigé en sanskrit.

5 Pour plus de détails sur les grammaires prakrites incomplètes/indirectes, voir Aussant (à paraître). 
décrire le sanskrit: l'Aștādhyāȳi engendre, au moyen d'un ensemble fini de règles, toutes les phrases correctes de la langue, et seulement elles. Dans ce genre de grammaire, l'enchaînement des règles s'arrête lorsqu'une séquence considérée comme correcte a été générée; rien n'empêche d'adjoindre, à la grammaire de départ, d'autres règles permettant d'obtenir d'autres séquences considérées comme correctes, y compris lorsque ces séquences sont celles d'une autre langue.

\subsection{Exemples de transfert de structure}

1.4.1 Des grammaires issues d'un transfert, certaines consistent, comme la plupart des grammaires du sanskrit ${ }^{6}$, en un ensemble de règles auquel s'adjoignent un ou plusieurs appendices :

- il peut s'agir d'un catalogue ou d'une grille de sons, comme dans le SCP, ce traité grammatical tibétain mentionné plus haut (cf. §1.1). Ainsi que le note Verhagen (2000b, p. 211 et 2001, p. 257 sq.), pour référer à des phonèmes spécifiques, le SCP recourt à une méthode qui associe des indications numériques à la grille de l'alphabet conventionnel tibétain. On peut représenter la grille des consonnes comme suit :

\begin{tabular}{l|l|l|l|l} 
& 1 & 2 & 3 & 4 \\
\hline sde $(=$ sanskrit $\operatorname{varga} a) 1$ & $\mathrm{k}$ & $\mathrm{kh}$ & $\mathrm{g}$ & $\mathrm{n}$ \\
\hline sde 2 & $\mathrm{c}$ & $\mathrm{ch}$ & $\mathrm{j}$ & $\mathrm{n}$ \\
\hline sde 3 & $\mathrm{t}$ & $\mathrm{th}$ & $\mathrm{d}$ & $\mathrm{n}$ \\
\hline sde 4 & $\mathrm{p}$ & $\mathrm{ph}$ & $\mathrm{b}$ & $\mathrm{m}$ \\
\hline sde 5 & $\mathrm{ts}$ & $\mathrm{tsh}$ & $\mathrm{dz}$ & $\mathrm{w}$ \\
\hline sde 6 & $\mathrm{z}$ & $\mathrm{z}$ & ' & $\mathrm{y}$ \\
\hline sde 7 & $\mathrm{r}$ & 1 & $\mathrm{~s}$ & $\mathrm{~s}$ \\
\hline sde 8 & $\mathrm{~h}$ & & & \\
\hline
\end{tabular}

Les phonèmes $/ \mathrm{g} /$ et $/ \dot{\mathrm{n}} / \mathrm{s}$ sont, dans ce contexte, identifiés comme «les deux derniers [éléments] du premier [sde]». Comme le remarque Verhagen (2001, p. 259), «This system is comparable in every respect to the approach we find in the

6 L'Aștâadhyāyi de Pānini, par exemple, consiste en un ensemble de près de 4000 règles appelé sūtrapātha («récitation des sūtra»), auquel sont adjoints, dès l'origine : 1) un Pratyāhārasūtra («aphorismes [relatifs aux] abréviations ») qui livre un inventaire ordonné des phonèmes du sanskrit; 2) un Dhätupātha («récitation des racines») qui fournit une liste de près de 2000 racines verbales divisée en dix classes; 3 ) un Ganapātha («récitation des groupes») qui rassemble 261 listes ordonnées d'unités linguistiques (thèmes nominaux, pronoms, numéraux, principalement). 
Kätantra tradition of Sanskrit grammar. The very first sütra of Kätantra states in fact that the traditional alphabet will serve as basis for reference to phonemes in this grammar. » La présentation des phonèmes dans la tradition pāṇinéenne est effectivement différente (cf. Cardona 1997, p. 80-84)-je reviendrai plus loin sur les différentes traditions ou écoles de grammaire sanskrite;

- il peut également s'agir d'un catalogue de racines verbales, sur le modèle du Dhātupātha de l'Aștâadhyāyī: c'est le cas pour le Kaccāyanavyākaraṇa, une grammaire du pali (en pali), composée au vI ${ }^{\mathrm{e}}$ ou vII s., à laquelle s'adjoint une Dhātumañjūsạ («Corbeille des racines»), ainsi que pour le Saddanītippakaraṇa d'Aggavamsa, autre grammaire du pali (XII ${ }^{\mathrm{e}} \mathrm{s}$.), à ceci près que, dans ce cas, la Dhātumālā («Guirlande des racines ») est (ou a été) intégrée à la grammaire (elle fait l'objet du chapitre 2 ; voir la partie consacrée au verbe dans la contribution de Nalini Balbir).

1.4.2 Voyons ce qu'il en est, maintenant, de l'arrangement des règles. L'Aștâadhyāy $\bar{l}$ est organisée en huit sections : les deux premières introduisent les notions fondamentales, la plupart des différentes classes de mots ainsi que les procédés techniques ; elles préparent, d'une certaine manière, l'application de la dérivation; les sections 3, 4 et 5 traitent de la dérivation des mots; les sections 6 , 7 et 8 présentent les résultats de la dérivation, les mots avec leurs jonctions internes et leurs accents (Pinault 1989, p. 371). Lorsqu'on aborde les règles dans le détail, on observe que celles qui doivent s'appliquer ensemble (i.e., les unes à la suite des autres, au cours de la dérivation d'une forme) se trouvent souvent dans des sections séparées par plusieurs règles, voire par plusieurs sections. Le fait est que l'ordre des règles est plus fréquemment gouverné par les fonctions des règles (général vs spécifique, interne vs externe, etc.), et par l'évitement de la répétition, que par les faits de langue décrits. Lorsque le besoin d'une grammaire pratique du sanskrit s'est fait sentir, cet ordre des règles a posé problème. La très grande majorité des grammaires post-pāṇinéennes du sanskrit ont opté pour un arrangement thématique des règles. Le Kätantra (IV ${ }^{\mathrm{e}} \mathrm{s}$. ?), par exemple, une grammaire du sanskrit écrite par un bouddhiste, s'organise comme suit:

1) section relative aux termes techniques, à la phonologie et à la phonétique;

2) section relative aux noms (thèmes nominaux, pronoms, cas sémantiques, emploi des désinences nominales, [composés, suffixes secondaires] ${ }^{7}$ );

3) section relative aux verbes (conjugaison, verbes dérivés, morphologie verbale, etc.);

4) section relative aux suffixes primaires.

À ma connaissance, lorsqu'une grammaire suit «l'ordre sanskrit des règles », il s'agit de l'ordre thématique, non de l'ordre sophistiqué de Pāṇini.

7 Les éléments figurant entre crochets seraient des ajouts tardifs. 
Les futures recherches menées sur la Grammaire Sanskrite Étendue devront permettre de mettre au jour les tendances générales concernant ce qui a été transféré : les termes métalinguistiques, les devises et les concepts ont-ils plus (ou moins) fait l'objet de transferts que la méthodologie ou la structure ? Y a-t-il eu des choix délibérés et ont-ils été explicités ${ }^{8}$ ? Ces choix ont-ils varié au cours de l'histoire? Pour quelles raisons?

\section{LES MODALITÉS DU TRANSFERT}

Comment les termes et les devises métalinguistiques, les concepts, les méthodes et les structures, initalement forgés pour la seule description du sanskrit, ont-ils été étendus à d'autres langues ? Répondre de façon précise à cette question implique nécessairement la conduite d'un certain nombre d'enquêtes sur le long terme. À ce stade des recherches, il me semble que l'on peut mentionner trois pistes de réflexion.

2.1 Première piste de réflexion : l'objet transféré a-t-il été transféré tel quel ou a-t-il été adapté (à la langue décrite et, quand elle existe, à une éventuelle description grammaticale propre, qui préexiste au modèle transféré)? La plupart des grammaires de langues «exotiques» écrites par des missionnaires chrétiens suivent le plan des grammaires gréco-latines, structuré en parties du discours (nom, verbe, participe, etc.) ; certaines de ces grammaires comportent des chapitres relatifs à telle ou telle partie du discours qui s'ouvrent sur le constat que cette partie n'existe pas dans la langue décrite (Auroux 1992, p. 39); ces formules, « il n’y a pas d'articles/d'adjectifs dans cette langue», indiquent que les auteurs de ces grammaires s'efforçaient de retrouver les parties du discours de la tradition grécolatine dans les langues « exotiques ». Ils avaient conscience de décrire des langues différentes et s'efforçaient d'exprimer ces différences - au moyen des outils dont ils disposaient-(Auroux 1992, p.39), leur perspective était clairement contrastive. Dans le contexte de la Grammaire Sanskrite Étendue, si on se limite à la terminologie métalinguistique (mais une étude de fond devra être menée sur l'ensemble des niveaux décrits plus haut), il semblerait que, dans la plupart des $\operatorname{cas}^{9}$ - je mets de côté les grammaires prakrites indirectes - il y a eu adaptation

8 Cf. Balbir (notice CTLF $\mathrm{n}^{\circ} 4340$ ) : «Les parties du discours et les notions grammaticales considérées par Mārkaṇdeya sont celles que reconnaît Pāninini [...] mais l'originalité de l'auteur est de dire clairement dans son premier aphorisme qu'il admet intégralement l'enseignement de son illustre prédécesseur à ce sujet (siddham pānniny-ādeh, I.1) alors que les autres grammairiens du prakrit ne se préoccupent nullement de la question et prennent ces données pour acquises. »

9 Un beau contre-exemple est fourni par Deven Patel (voir sa contribution dans ce numéro): l'emploi du terme tumUN-anta (qui signifie littéralement «qui se termine par tum» et qui désigne l'infinitif sanskrit, tel gantum «aller» de la racine gam-) dans l'Āndhraśabdacintāmaṇi (grammaire du télougou rédigée en sanskrit), pour désigner également l'infinitif. Or, en télougou, le suffixe d'infinitif est $k u$, non tum. 
de la terminologie technique sanskrite ${ }^{10}$. Cette adaptation a avant tout consisté en une sélection de termes techniques : seuls les termes pertinents pour la description ont été retenus. Les grammaires composées en sanskrit s'en sont généralement tenues à cette forme d'adaptation (certaines ont parfois complété la terminologie issue du transfert, pour rendre compte de traits qu'elle ne couvrait pas, tel l'Āndhraśabdacintāmaṇi, grammaire du télougou (voir la contribution de Deven Patel $^{11}$ ); les grammaires composées dans des langues autres que le sanskrit, comme le tamoul, le pali, le tibétain, sont généralement allées plus loin dans l'adaptation de la terminologie et ont doublé la sélection des termes d'une appropriation de ces termes:

sur le plan formel :

- certaines ont adapté des termes sanskrits à la phonétique de la langue de description ; on trouve, par ex., dans le Vīracōliyam, une grammaire du tamoul rédigée en tamoul au $\mathrm{XI}^{\mathrm{e}} \mathrm{s}$. par un grammairien tamoul bouddhiste - Puttamittiranāar- les termes canti et tātu qui sont les formes tamoulisées de sandhi et dhātu;

- certaines ont traduit littéralement («traduction calque ») des termes sanskrits dans la langue de description; Verhagen (2001, p. 216) donne l'exemple du tibétain byed-pa, calque du sanskrit karaṇa, avec ce même double emploi technique: «instrument de réalisation de l'action» (i.e., cas sémantique marqué par l'instrumental) et « instrument de réalisation des phonèmes » (i.e., point d'articulation);

- certaines ont donné des équivalents des termes sanskrits, ainsi ticaic-col (litt. «mot régional») et vața-col (litt. « mot du Nord») seraient les équivalents tamouls respectifs de deśi (litt. «local», « régional», mots indépendants du sanskrit, probablement empruntés à des dialectes régionaux) et tatsama (litt. «pareil à cela », i.e. les mots sanskrits).

parfois aussi sur le plan du contenu : voir l'exemple donné en 1.2 de la classification de l'objet grammatical repris par Cēnāvaraiyar - la classification élaborée par Bhartṛhari n'a pas été transférée telle quelle, elle a fait l'objet d'une adaptation fine - ainsi que la notion de punar-nilai étudiée dans la contribution de Giovanni Ciotti.

2.2 Deuxième piste de réflexion : peut-on retracer le cheminement, l'itinéraire du transfert? Il faut se rappeler que plusieurs écoles de grammaire se sont développées

10 Verhagen (2001, p. 223) fournit quelques contre-exemples tirés du SCP et du TKJ.

11 Cf. Verhagen (2001, p. 220-221) pour des exemples dans le SCP et le TKJ et Ananthanarayana (2017, p. 83) pour des exemples dans le Bālavyākaraṇamu de Paravastu Cinnayasūri, une grammaire du télougou publiée en 1858 . 
en Inde. On peut classer ces écoles en deux catégories : l'école pāṇinéenne et les écoles non-pāninéennes. Le fait est que la pensée grammaticale indienne traditionnelle a été très profondément marquée par l'Așțādhyāyī de Pāṇini, ouvrage que l'on présente, en Inde comme en Occident, comme le texte fondateur du Vyākaraṇa. Durant les siècles qui ont suivi la composition de cette oeuvre (vers le $\mathrm{v}^{\mathrm{e}} \mathrm{s}$. av. n. ère), certains grammairiens ont décidé de suivre fidèlement la méthode et les techniques pāṇinéennes, créant ainsi «l'école pāṇinéenne », tandis que d'autres s'en sont plus ou moins éloignés (ou ont été considérés, par les pāṇinéens, comme s'en étant éloigné). Cette distanciation par rapport au modèle pāṇinéen s'explique principalement par le besoin d'une grammaire plus pratique, plus pédagogique ( $d$ 'où l'organisation thématique des règles, que l'on a vue précédemment) ${ }^{12}$. Pour autant que l'on sache, les grammaires sanskrites organisées thématiquement sont apparues au sein des communautés bouddhistes et jaïnes (donc, en dehors de l'école pāṇinénne, qui est étroitement associée au brahmanisme), après l'adoption de versions sanskrites de leurs textes canoniques (ou de certains de leurs textes canoniques). Le Kātantra («Petit traité ») de Śarvavarman, que j'ai eu l'occasion de mentionner plus haut, constitue la plus ancienne tentative d'organisation thématique de règles grammaticales que nous connaissons (IV ${ }^{\mathrm{e}} \mathrm{s} . ?$ ). Cette grammaire, qui est peut-être la réfection d'une grammaire plus ancienne - le Kaumāralāta (cf. Scharfe 1977, p. 162) - a influencé plusieurs grammaires ultérieures, parmi lesquelles la grammaire palie de Kaccāyana (Kaccāyanavyākaraṇa, ou Sandhikappa, vi ${ }^{\mathrm{e}}-\mathrm{VII}^{\mathrm{e}} \mathrm{s}$.) ainsi que le ŚSabdānuśāsana, grammaire du jaïn Hemacandra, qui couvre le sanskrit et les prakrits. Une autre grammaire organisée en sections thématiques, le Cāndravyākaraṇa («La grammaire de Candra»), elle aussi composée par un lettré bouddhiste - Candragomin - qui aurait vécu durant le $\mathrm{v}^{\mathrm{e}} \mathrm{s}$., a joué un rôle similaire. Le Cāndravyākaraṇa, première réfection d'ampleur du traité pāṇinéen, est la grande grammaire des bouddhistes: elle a beaucoup circulé et a été principalement préservée dans les lieux où le bouddhisme a essaimé (Népal, Tibet, Birmanie, Sri Lanka). La postérité du Kātantra et du Cāndravyākaraṇa montre que le transfert des «outils» élaborés pour le sanskrit à des langues autres que le sanskrit, ne s'est pas fait seulement et directement à partir du modèle pāṇinéen. Le transfert a parfois emprunté des voies complexes, où s'entremêlent plusieurs «modèles »: ainsi, la littérature grammaticale relative au pali, bien qu'elle soit en partie autonome, révèle l'influence de l'Aștâadhyāy $\bar{l}$, du Kātantra et du Cāndravyākaraṇa mais également d'autres textes brahmaniques grammaticaux (Ruiz-Falqués 2014, p.390 et 392). À son tour, le modèle grammatical élaboré pour le pali a été «étendu» au birman (Kasevitch 2000,

12 Une divergence d'opinion concernant des points de théorie précis (telle que la description de la formation des vrtti; voir Cardona 2008 à ce sujet) a également joué un rôle. 
p. 183). Les contributions de Giovanni Ciotti, Victor D'Avella et Deven Patel mentionnent d'autres exemples.

2.3 Troisième piste de réflexion: tout transfert est étroitement lié aux sujets qui l'opèrent, comme nous le rappelle Sylvain Auroux (1992, p.35). Ce dernier souligne l'importance que revêt la situation de ces sujets, " selon qu'ils sont ou non des locuteurs natifs de la langue vers laquelle a lieu le transfert» et distingue, respectivement, l'endo-transfert (les sujets opérant le transfert sont des locuteurs natifs de la langue décrite) de l'exo-transfert (les sujets opérant le transfert ne sont pas des locuteurs natifs de la langue décrite). Cette distinction est-elle opératoire, dans le contexte de la Grammaire Sanskrite Étendue ? Ce n'est pas évident, tout au moins sous cette formulation. À date ancienne, il est quasiment impossible de savoir de quelle(s) langue(s) tel grammairien était locuteur natif et, quand bien même on le saurait, les langues que l'on décrit alors sont soit des langues « sacrées », telles le sanskrit ou le pali (respectivement associées au Canon védique ou bouddhique), soit des langues littéraires, telles les prakrits et le tamoul poétique. Si l'on met de côté quelques rares exceptions ${ }^{13}$, les grammaires composées dans l'Inde ancienne ne portent pas sur des langues «maternelles »-c'est, du moins, ce que laissent penser les documents qui nous sont parvenus. En outre, à cette époque, le plurilinguisme constituait la norme, et il n'est peut-être pas inconcevable d'imaginer que les grammairiens recouraient à plusieurs «langues de travail».

Ceci étant dit, on peut peut-être voir, dans l'élaboration de la pensée grammaticale relative au pali, au tamoul, comme dans celle relative au tibétain, une forme d'endo-grammatisation: si ces trois langues ont été décrites, dans une certaine mesure, via des outils élaborés pour le sanskrit, elles ont donné lieu, vraisemblablement avant la dissémination de ces outils, à une réflexion propre, réflexion qui s'est exprimée en pali, en tamoul ou en tibétain, non en sanskrit. L'endo-grammatisation peut également être utile pour décrire l'apparition, au début de la seconde moitié du premier millénaire, des grammaires de vernaculaires ou «langues régionales», telle l'Āndhrabhāṣâhhūṣaṇamu, la première (?) grammaire du télougou rédigée en télougou au XII ${ }^{\mathrm{e}} \mathrm{s}$. par Ketana ou le Śabdasmṛti de Nāgavarma, grammaire du kannaḍa rédigée en kannaḍa au XII ${ }^{\mathrm{e}} \mathrm{s}$. également. Les recherches à venir nous le diront.

$13 \mathrm{La}$ grammaire de Pānini formule des règles qui valent, en théorie, pour deux variétés linguistiques: la variété commune dite bhạṣā (litt. "parole») "langue parlée», qui correspond à la forme classique de la langue sanskrite parlée au nord du sous-continent, et celle dite chandas (litt. « mètre » puis « texte versifié ») « littérature sacrée », qui correspond à une forme ancienne de la langue, conservée dans les textes védiques. De même, certaines grammaires tamoules décrivent, à côté du tamoul poétique, le tamoul parlé par les catégories supérieures de la population (valakku, cf. Chevillard notice CTLF 4351). 
Quoi qu'il en soit, le problème subsiste avec certaines grammaires tardives : au $\mathrm{XVI}^{\mathrm{e}}$ s., Kṛ̣ṇadāsa, probablement membre d'une communauté de brahmanes d'origine persane (les brahmanes Maga), est chargé, par l'empereur moghol Akbar, de rédiger une grammaire et un glossaire du persan tel qu'il est alors parlé en Inde. Cette grammaire de 480 règles - le Pārasīprakāśa -, rédigée en sanskrit, consiste essentiellement en opérations de substitution, à l'instar des grammaires prakrites. S'agit-il d'un cas d'exo-grammatisation (l'auteur est brahmane, la grammaire est en sanskrit, elle suit le modèle sanskrit, elle porte sur une langue étrangère) ou d'endo-grammatisation (l'auteur est d'origine persane, il décrit sa langue au moyen d'un modèle grammatical élaboré pour une langue autre qu'elle)? Selon les spécialistes qui ont travaillé sur cette grammaire (notamment Scharfe 1977, p. 196197), cet ouvrage ne pouvait servir à apprendre le persan aux lettrés indiens formés en sanskrit, il s'agissait plus de démontrer que le persan, langue de la Cour de Delhi, pouvait être traité comme un transfert du sanskrit, à l'égal des prakrits. Ce cas de figure (il y en a peut-être d'autres) ne se laisse vraisemblablement pas aborder en termes d'exo- ou d'endo-grammatisation.

\section{LES CAUSES DU TRANSFERT}

Comme le rappelle Sylvain Auroux (1992, p.21), la première cause de grammatisation est le besoin d'apprentissage d'une langue étrangère, qui peut répondre à divers intérêts pratiques, tels que l'accès à une langue d'administration, l'accès à un corpus de textes sacrés, l'accès à une langue de culture, les relations commerciales et politiques, l'importation/l'exportation d'une doctrine religieuse. La seconde cause de grammatisation concerne la politique d'une langue donnée, qui peut se traduire par l'organisation et la régulation d'une langue littéraire. On retrouve, sans surprise, ces différents intérêts pratiques en Asie et, notamment, en Inde. Les outils élaborés, à date très ancienne, pour la description du sanskrit, étaient particulièrement aboutis et d'une très grande puissance descriptive. Ils ont tout naturellement permis, en étant transférés à d'autres langues, de répondre à ces intérêts pratiques. Mais il y a plus, comme le rappelle le Pārasīprakāśa, cette grammaire du persan, rédigée en sanskrit, destinée à démontrer que le persan pouvait être traité comme un transfert du sanskrit: décrire une langue au moyen d'outils élaborés pour le sanskrit, c'est créer une relation avec la langue sanskrite elle-même et, partant, avec le prestige qui lui est associé, avec la culture et les doctrines dont elle a été le véhicule (voir la contribution de Deven Patel pour un autre exemple).

Il se trouve que dans plusieurs cas, la relation entre le sanskrit et la langue à décrire est fondée linguistiquement: les variétés d'indo-aryen moyen et d'indoaryen moderne sont historiquement liées au sanskrit ou, tout au moins, à l'indoaryen ancien. La proximité typologique de ces langues a tout naturellement facilité 
le transfert des outils (et, inévitablement, le transfert du prestige de la langue sanskrite). Dans d'autres cas, si les langues ne sont pas liées historiquement, elles peuvent contenir un nombre significatif de mots d'origine sanskrite, c'est le cas du malayalam et du vieux-javanais, notamment; là encore, la présence d'un vocabulaire sanskrit dans ces langues peut justifier le transfert (des outils/du prestige).

Le Pārasīprakāśa montre que les outils élaborés pour le sanskrit ont également été transférés à une langue (le persan) dont la relation au sanskrit est nettement moins évidente linguistiquement; dans ce cas précis, c'est la quête d'une légitimité politique qui est visiblement recherchée (le persan est l'égal des prakrits puisqu'il peut être décrit comme eux). Il y a sans doute d'autres cas similaires à celui-ci.

Si les descriptions grammaticales élaborées pour le sanskrit ont joué un rôle majeur dans l'histoire de la grammatisation de diverses langues, c'est sans aucun doute parce-qu'elles associent un modèle descriptif incroyablement sophistiqué à une langue auréolée de prestige (langue de différents textes sacrés - brahmanisme, bouddhisme-, langue d'une immense littérature savante, langue cosmopolite, au premier millénaire, d'un espace qui semble sans limite, cf. Pollock 2007). Le transfert des outils élaborés pour le sanskrit c'est, d'une manière ou d'une autre, le transfert de l'incroyable puissance d'un modèle grammatical et/ou le transfert d'un prestige incommensurable. Ce transfert s'est réalisé de manières diverses, à des fins diverses; il nous faudra plusieurs années, et un collectif fédérant des compétences variées, pour en dresser la cartographie.

\section{BiBLIOGRAPHIE}

\section{Sources primaires}

Aggavamısa. Saddanīti, La grammaire palie d'Aggavamsa. Texte établi par Helmer Smith, Lund, C.W.K. Gleerup, 1928-66.

Kaccāyana. Kaccāyanavyākaraṇa ed. by L.N. Tiwari and B. Sharma, Varanasi, Tara Publications, 1962.

Kṛ̣ṇadāsa. Über den zweiten, grammatischen, Pārasīprakāça des Kṛishṇadāsa von Albrecht Weber, Berlin, Verlag der Königl. Akademie der Wissenschaften, 1889.

Pāninin. La grammaire de Pạnini. Texte sanskrit, traduction française avec extraits des commentaires par Louis Renou, 2 vols., Paris, Bibliothèque de l'École française d'Extrême-Orient, 1966.

Purușottama. Le Prākrtānnuśāsana de Purușottama par Luigia Nitti-Dolci, Paris, Cahiers de la Société Asiatique, 1938.

Puttamittiran. Vìracōliyam mūlamum Peruntēvanār iyarriya uraiyum ed. by K.R. Kōvintarāja Mutaliyār, Madras, Pavānantar Kalakam, 1942 (réimpression : Tinnevelly, 1970).

Śarvavarman. The Kātantra-Vyākaraṇa ed. by R.S. Saini, Delhi-Varanasi, Bharatiya Vidya Prakashan, 1987.

Vararuci. The Prākrita-Prakāsa or The Prākrịt Grammar of Vararuchi with the commentary (Manoramā) of Bhämaha - With the text, notes, English translation, and index of Prākṛt words by E.B. Cowell, Calcutta, Punthi Pustak, 1962 ( $1^{\text {re }}$ éd. : Hertford, 1854). 


\section{Sources secondaires}

Ananthanarayana, H.S., 2017. «Pāṇinian model for describing Telugu in Bālavyākaranamu», International Journal of Dravidian Linguistics 46.1, 70-85.

Auroux, Sylvain, 1992. "Introduction. Le processus de grammatisation et ses enjeux ", Auroux, S. (éd.), Histoire des idées linguistiques, tome 2 (Le développement de la grammaire occidentale), Liège, Mardaga, 11-64.

- 1994. La révolution technologique de la grammatisation, Liège, Mardaga.

Aussant, Émilie, à paraître. « Les grammaires prakrites : des 'Transfer Grammars' avant l'heure ? ", Actes du $1^{\text {er }}$ Congrès mondial de la traductologie (Paris, 2017).

Balbir, Nalini, Notice « Mārkaṇdeya » (n4340) du CTLF (http://ctlf.ens-lyon.fr).

Cardona, George, 1997. Pānini. His work and its traditions, volume I: Background and Introduction, $2^{\text {nd }}$ revised and completed edition, Delhi, Motilal Banarsidass ( $1^{\text {re }}$ éd. : 1988).

- 2008. «Theoretical Precedents of the Kātantra», Kaul, M. et Aklujkar, A. (eds.), Linguistic Traditions of Kashmir, Essays in Memory of Pandit Dinanath Yaksha, New Delhi, D.K. Printworld, 300-367.

Chevillard, Jean-Luc, Notice « Tolkāppiyanār » (n4351) du CTLF (http://ctlf.ens-lyon.fr).

Kasevitch, Vadim Borisovich, 2000. «Indian influence on the linguistic tradition of Burma», Auroux, S., Koerner, E.F.K, Niederehe, H.-J. et Versteegh, K. (eds.), History of the Language Sciences, An International Handbook on the Evolution of the Study of Language from the Beginnings to the Present, vol. I, Berlin/New York, Walter de Gruyter, 182-186.

Miller, Roy Andrew, 2000. «The Establishment of Tibetan Linguistics», Auroux, S., Koerner, E.F.K, Niederehe, H.-J. et Versteegh, K. (eds.), History of the Language Sciences, An International Handbook on the Evolution of the Study of Language from the Beginnings to the Present, vol. I, Berlin/New York, Walter de Gruyter, 203-206.

Ojihara, Yutaka, 1971. " Un chapitre de la Saddanīti comparé aux données pāṇinéennes », Journal asiatique 279, 83-97.

Pinault, Georges-Jean, 1989. «Le système de Pānini », Auroux, S. (ed.), Histoire des idées linguistiques, tome 1 (La naissance des métalangages en Orient et en Occident), Liège, Mardaga, 371-400.

Pollock, Sheldon, 2007. The language of the Gods in the world of men, Sanskrit, culture and power in premodern India, New Delhi, Permanent Black.

Ruiz-Falqués, Aleix, 2014. «The creative erudition of Chapața Saddhammajotipāla, a $15^{\text {th }}$ Century grammarian and philosopher from Burma», Journal of Indian Philosophy 43, 389-426.

Scharfe, Hartmut, 1977. Grammatical Literature (A History of Indian Literature II.2). Wiesbaden, Otto Harrassowitz.

Vergiani, Vincenzo, 2013. "The adoption of Bhartrhari's classification of the grammatical object in Cēnāvaraiyar's commentary on the Tolkāppiyam», Cox, W. et Vergiani, V. (eds.), Bilingualism and cross-cultural fertilisation: Sanskrit and Tamil in medieval India, Pondicherry, Institut français de Pondichéry \& École française d'Extrême-Orient, 161-197.

Verhagen, Pieter Cornelius, 2000a. «The classical Tibetan grammarians», Auroux, S., Koerner, E.F.K, Niederehe, H.-J. et Versteegh, K. (eds.), History of the Language Sciences, An International Handbook on the Evolution of the Study of Language from the Beginnings to the Present, vol. I, Berlin/New York, Walter de Gruyter, 207-210.

- 2000b. "The influence of the Sanskrit tradition on Tibetan indigenous grammar», Auroux, S., Koerner, E.F.K, Niederehe, H.-J. et Versteegh, K. (eds.), History of the Language Sciences, An International Handbook on the Evolution of the Study of Language from the Beginnings to the Present, vol. I, Berlin/New York, Walter de Gruyter, 210-214.

- 2001. A History of Sanskrit Grammatical Literature in Tibet, vol. II (Assimilation into Indigenous Scholarship), Leiden, E.J. Brill Co, Handbuch der Orientalistik. 